\title{
Reformation de surfaces de matériaux métalliques par laser à excimères ou laser à vapeur de cuivre
}

\author{
B. Esmiller et V. Baudinaud
}

AEROSPATIALE, Département Techniques de Base Physique et Mathématiques, Service Effets des Lasers sur la Matière, Centre Commun de Recherches Louis Blériot, 12 rue Pasteur, 92152 Suresnes cedex, France

\begin{abstract}
Résumé : Cet article présente les travaux de reformation de surface par laser pulsé (laser excimère ou laser à vapeur de cuivre) de matériaux métalliques (alliages d'aluminium : AS7 et A12024, aciers inoxydables : Marval X12 et Z10CNT18.11, fonte lamellaire ou sphéroïdale), couramment utilisés dans les industries aéronautiques et automobiles, en vue d'améliorer certaines propriétés (résistance à la corrosion, résistance à l'usure ou au frottement).
\end{abstract}

\section{INTRODUCTION}

Le développement de nouvelles sources laser émettant dans l'ultraviolet comme les lasers à excimères a permis d'envisager de nouvelles applications, en particulier dans les industries aéronautiques ou automobiles. Cet article présente donc les travaux réalisés au Centre Commun de Recherches LouisBlériot d'Aerospatiale en collaboration avec PSA, l'Ecole des Mines, le CEA et I'IRPHE sur le thème reformation de surfaces de matériaux métalliques par laser excimère ou par laser à vapeur de cuivre. Ces études ont pour objectifs de comprendre les mécanismes d'interaction laser-matériau et d'évaluer les potentialitês des lasers excimères pour le traitement de surface afin de développer à terme de nouveaux procédés. Dans le cadre de cette étude, suivant les applications envisagées, le traitement de surface vise à améliorer certaines propriétés telles que :

- la modification des critères d'état de surface (rugosité),

- la résistance à la corrosion pour l' $\mathrm{Al} 2024$,

- les propriétés tribologiques pour l'AS7 et la fonte,

- la résistance à l'usure sans détérioration de la résistance à la corrosion pour les aciers

inoxydables, Marval X12 et Z10CNT18.11.

\section{TRAITEMENT DE SURFACES PAR LASER PULSE}

Le rayonnement laser est un vecteur d'énergie particulièrement bien adapté à la réalisation de traitement de surface de type thermique pour différentes raisons :

- le transfert d'énergie au matériau est beaucoup plus rapide que par les procédés

conventionnels

- les zones traitées sont limitées et parfaitement localisées

- pas de contact entre l'outil et le matériau

- l'impact sur l'environnement est limité (le laser serait alors proposé en remplacement de traitements chimiques polluants).

L'utilisation de lasers comme le LVC et le laser à excimère dans cette application est une idée relativement neuve, le traitement de surface étant surtout réservé aux lasers continus $\left(\mathrm{CO}_{2}\right)$. Ces lasers pulsés apportent les avantages suivants :

- l'absorption des matériaux est plus importante dans les faibles longueurs d'onde,

- les courtes durées d'impulsion permettent des effets thermiques limités aux zones choisies, et sur des profondeurs faibles (ce qui conserve au matériau ses propriétés en volume). 
Les lasers à excimère et les LVC sont caractérisés par des impulsions très courtes (quelques dizaines de ns) et de haute énergie. Avec une intensité suffisante, la fusion et la vaporisation du matériau est rapidement atteinte, sur une profondeur de l'ordre de quelques $\mu \mathrm{m}$. Il s'agit de provoquer une fusion superficielle du matériau, qui sera suivie d'une trempe ultra-rapide. Les zones traitées sont très localisées, et les ZAT très limitées (la diffusion thermique est stoppée par le refroidissement très brutal). Il résulte de ce traitement de nouvelles propriétés surfaciques pour les matériaux (état de surface, affinement des grains, modification chimique.

\section{TRAVAUX DE REFORMATION DE SURFACES}

Les caractéristiques des lasers utilisés sont reportées dans le tableau suivant :

\begin{tabular}{|l|c|c|c|}
\hline & laser excimère & laser excimère & $\begin{array}{c}\text { laser à vapeur } \\
\text { de Cuivre }\end{array}$ \\
\hline localisation & IRPHE & AEROSPATLALE & CEA \\
\hline $\begin{array}{l}\text { longueur d'onde } \\
\text { (gaz) }\end{array}$ & $308 \mathrm{~nm}$ & $\begin{array}{c}248 \mathrm{~nm} \\
\mathrm{KrF}\end{array}$ & $\begin{array}{c}510 \mathrm{~nm} \\
\text { et } 578 \mathrm{~nm}\end{array}$ \\
\hline énergie/pulse & $\mathrm{XeCl}$ & $250 \mathrm{~mJ}$ & $20 \mathrm{~mJ}$ \\
\hline fréquence maximale & $300 \mathrm{~mJ}$ & $350 \mathrm{~Hz}$ & $5.6 \mathrm{kHz}$ \\
\hline puissance maximale & $250 \mathrm{~Hz}$ & $90 \mathrm{~W}$ & $100 \mathrm{~W}$ \\
\hline durée d'impulsion & $75 \mathrm{~W}$ & $25 \mathrm{~ns}$ & $55 \mathrm{~ns}$ \\
\hline
\end{tabular}

Le dispositif utilisé est présenté figure 1. Un masque a étế utilisé afin d'obtenir une tache laser dont la répartition spatiale énergétique est homogène. Pour le laser à vapeur de cuivre, l'utilisation d'une fibre optique pour le transport permet l'homogénéisation du faisceau laser.

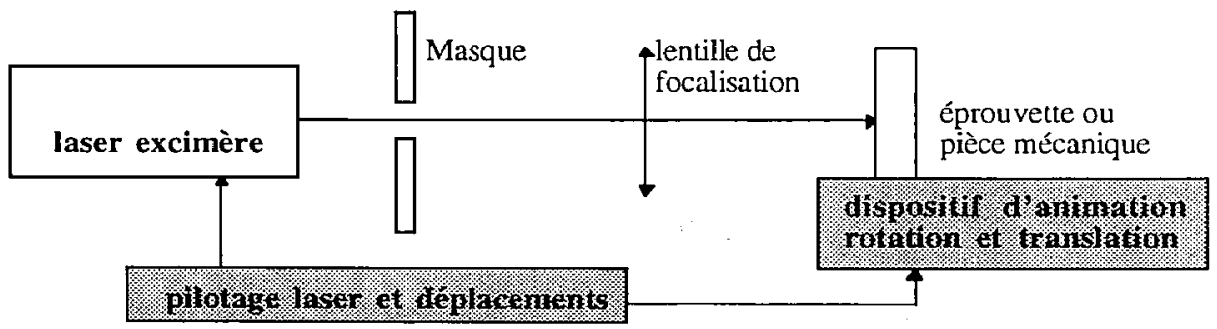

Figure 1 : dispositif experimental

les conditions d'irradiations sont les suivantes:

- densité d'énergie, entre $0,5 \mathrm{et} 4 \mathrm{~J} / \mathrm{cm}^{2}$

- durée d'impulsion : entre 25 et $55 \mathrm{~ns}$

- recouvrement : entre 4 et 32 tirs par point

- traitement réalisé sous air

\section{CARACTERISATION DES MATERIAUX}

Afin de caractériser la surface et les propriétés des matériaux, avant et après traitement laser, différents moyens d'analyses ont été utilisés.

L'observation visuelle réalisée à l'aide d'un microscope électronique à balayage (MEB) de la surface du matériau apporte nombre d'informations utiles sur le degré de polissage de la surface, les conséquences visibles du traitement (fusion, mouvements du bain liquide, bourrelets...), le changement de structure du matériau (affinement des grains...).

Dans de nombreuses applications industrielles, la rugosité des matériaux doit être contrôlée (pour les pièces de frottement, pour les collages...). Il faut donc quantifier ce paramètre lors du traitement laser. 
Il a êté montré que le traitement laser, selon la rugosité de départ, la fluence et le nombre de tirs, pouvait avoir un effet de lissage de la surface ou au contraire augmenter la rugosité.

Les analyses tribologiques concernent le comportement en usure et frottement du matériau, pour un système tribologique donné. Ces paramètres sont mesurés à l'aide d'un tribomètre pion-disque. L'essai consiste à faire glisser sans roulement une bille de $3 \mathrm{~mm}$ de diamètre (le pion) sur la surface de l'échantillon. Ces essais ne sont pas représentatifs des conditions réelles d'utilisation des pièces (notamment pour les chemises de cylindres, même si le frottement sec au démarrage dans le moteur s'apparente au frottement étudié lors des essais). Le tribomètre est un outil permettant de mettre en évidence certains phénomènes, de les expliquer et d'établir des comparaisons entre les différentes conditions de traitement.

L'analyse est effectuée au moyen d'une microsonde électronique. Le principe consiste à irradier la surface du matériau par un faisceau d'électrons qui provoque une émission de rayons $\mathrm{X}$ caractéristiques des éléments qui composent le matêriau.

La dureté, caractérisant la résistance du matériau à la déformation, est mesurée par un essai en pénétration dans le matériau.

Une partie des échantillons est destinée à être exposée au brouillard salin (en général 700 heures) pour évaluer leur résistance à la corrosion. L'attaque accélérée corrosive par un brouillard salin artificiel, de composition définie (concentration de la solution saline : $5 \%$ ) est réalisée dans des conditions précises de température $\left(35^{\circ} \mathrm{C} \pm 1^{\circ} \mathrm{C}\right.$ ), de pression $(1$ bar $\pm 0,2)$ et de $\mathrm{pH}(7$ entre 6,5 et 7,2$)$. Dans le cadre de cette étude, pour chacun des matériaux, de nouvelles échelles de gravité ont été définies pour analyser l'état de corrosion car il n'est pas possible, pour ce type de traitement, de comparer les résultats avec des échelles normalisées.

\section{RESULTATS}

\subsection{Traitement des alliages d'aluminium}

\subsubsection{AS7}

L'AS7 est un alliage d'aluminium utilisé pour fabriquer des étriers de frein (PSA) ayant une forte concentration en silicium $(6,5$ à $7,5 \%)$. Il a été obtenu une légère diminution du coefticient de frottement (figure 2) pour toute la gamme de fluence étudiée et ceci pour un faible nombre de cycles $(<80)$. Les fortes fluctuations du coefficient de frottement en fonction du nombre de tours, aussi bien sur l'échantillon traité que brut, proviennent des nombreuses porosités présentes à la surface des éprouvettes. Il est impossible, en raison de leur taille de les éliminer à l'aide d'un traitement laser. Cette amélioration provient de l'affinement des grains qui permet une augmentation de la microdureté. La figure 3 montre une forte augmentation des contraintes résiduelles, diminuant ainsi la tenue en fatigue.

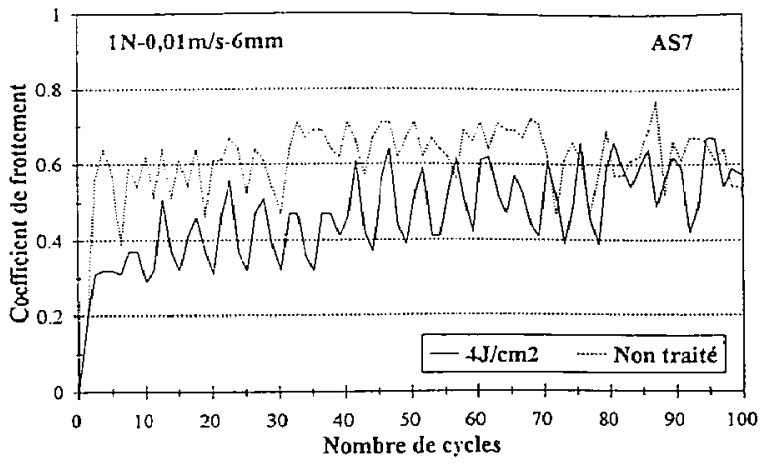

Figure 2 : evolution du coefficient de frottement pour une eprouvette en AS7 traitee à $4 \mathrm{~J} / \mathrm{cm}^{2}$ 


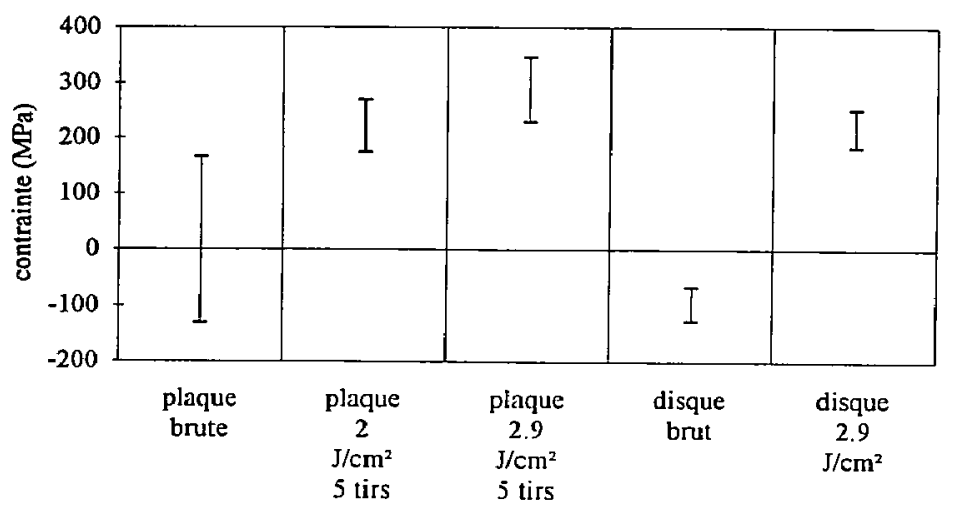

Figure 3 : contraintes résiduelles de l'AS7 avant et après traitement laser

\subsubsection{Al 2024}

Cette alliage d'aluminium est très utilisé dans l'aéronautique. Actuellement, la protection contre la corrosion du 2024T351 est assurée par une anodisation non colmatée suivie d'une peinture. Ces propriétés intrinsèques lui permettent une bonne résistance à la corrosion évaluée à 3000 heures en Immersion-Emersion Alternée sur des éprouvettes dont la protection a été rayée jusqu'au métal de base. Pour des densités d'énergie supérieures ou égales à $2 \mathrm{~J} / \mathrm{cm}^{2}$, il se forme, au cours des premières heures d'exposition au brouillard salin, en surface des éprouvettes traitées une fine couche d'oxyde homogène. Cette couche passive le matériau et par la suite, il n'a pas été observé d'évolution de la corrosion du matériau. Après 350 heures d'exposition cet alliage n'est pas corrodé et après 700 heures quelques taches et coulures sont observées, cependant un mois après l'arrêt d'exposition les piqûres de corrosion sont observées sur la surface (figure 4). Pour ces conditions de traitement laser, il n'y a pas apparition de piqûres comme sur les éprouvettes brutes ou traitées avec une densité d'énergie insuffisante pour obtenir une fusion superficielle du métal.

Echelle de corrosion de 1'Al 2024

1 : surface homogène ("passivée")

2 : présence de coulures

3 : taches noires associées aux coulures

$4:$ extension des taches et des coulures

$5:$ taches et coulures majoritaires en surface

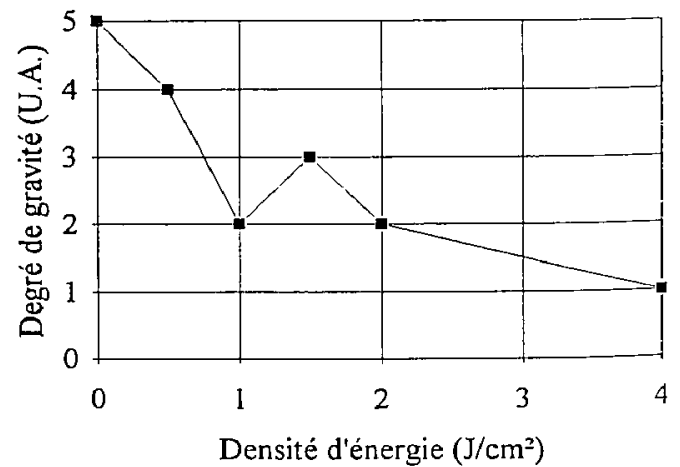

Figure 4: Al 2024 irradié par laser excimère $(\mathrm{KrF})$, évolution de degré de corrosion en fonction de la fluence

Le spectre de diffraction $X$ de l'alliage traité ne fait pas apparaître de nouveaux pics par rapport à celui de l'échantillon brut (figure 5). Il n'y a donc pas création de nouvelles phases dont la teneur est supérieure à $1 \%$. En revanche, la largeur des pics montre traduit un éventuel affinement du grain lors du traitement et la position relative des pics, l'apparition de contraintes résiduelles en traction qui peut 
entraîner une décohésion de la couche traitée. Des mesures de contraintes résiduelles ont permis de confirmer ce résultat (contraintes résiduelles en traction des échantillons traités par laser proches de la limite d'élasticité du matériau). D'autre part des essais de micro-indentation ont permis de vérifier la décohésion de la couche traitée (figure 6). Il a donc été obtenu une nette amélioration de la tenue à la corrosion mais une diminution des propriétés mécaniques (tenue en fatigue, dureté,...).

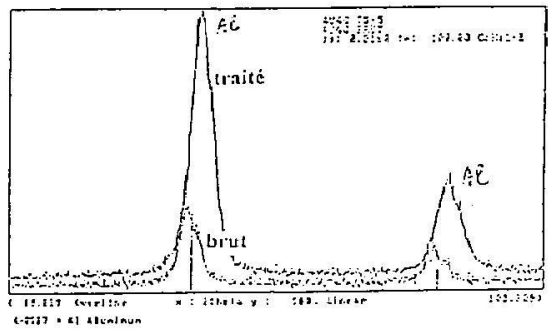

Figure 5: Al2024 inadié par laser excimère, spectre de diffraction $\mathrm{X}$ de la couche refondue

\subsection{Traitement des aciers inoxydables}

Les compositions chimiques des deux inox sont données dans le tableau ci-dessous.

\begin{tabular}{|c|c|c|c|c|c|c|c|}
\hline & $\mathbf{C}$ & $\mathbf{C r}$ & $\mathbf{N i}$ & Mo & Ti & Al & Fe \\
\hline Z10CNT 18-11 & 0,08 & 18,00 & 12,00 & & $>5 \mathrm{C}$ & & balance \\
\hline Marval X12 & 0,02 & 12,16 & 9,1 & 2,00 & 0,35 & 0,59 & balance \\
\hline
\end{tabular}

L'acier inoxydable Z10CNT18-11 présente une structure austénitique eandis que le Marval X12 (EZ1CND12 09) est martensitique. Ces aciers inoxydables sont utilisés en aéronautique pour des pièces de fixation. Le but de traitement laser est d'améliorer les propriétés tribologiques sans dégrader la résistance à la conosion.

\subsubsection{Tests de tenue à la corrosion}

Actuellement, une décontamination de la surface (élimination des particules ferreuses) ou l'élimination (ponçage, décapage chimique) de la couche déchromisée en cas de traitement thermique suffit à assurer une tenue en corrosion de plusieurs milliers d'heures en brouillard salin sans apparition de corrosion. En général pour le Marval X12, le traitement laser entraine une forte dégradation de la résistance à la corrosion par rapport à l'éprouvette non irradiée et ceci pour toutes les densités d'énergie (figure 7). 
Echelle de corrosion du MARVAL X12

1 : surface vierge

2 : apparition d'auréoles

3 : points sombres épars

4 : début d'oxydation autour des points

5 : apparition des taches de corrosion

$6:$ extension des taches

$7:$ taches très étendues

8 : coalescence de taches de corrosion

9 : corrosion de surface $(>80$

$\%)$

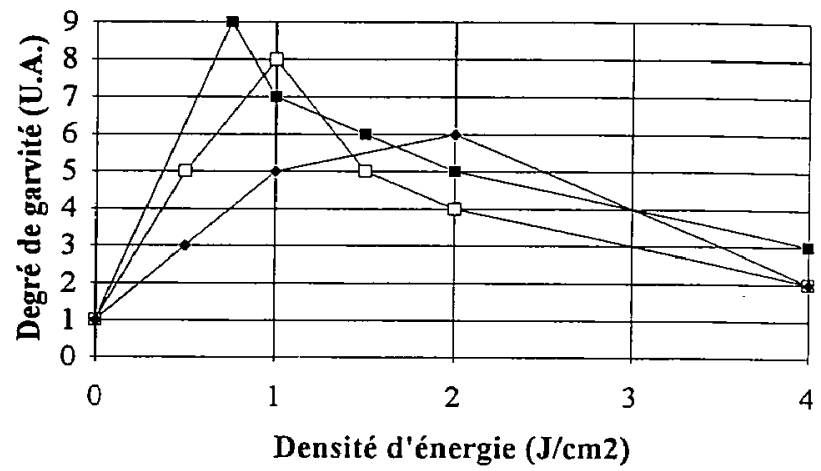

10 : corrosion généralisée

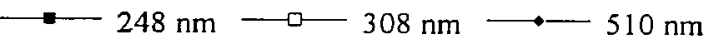

Figure 7 : Marval X12 irradié par laser, évolution de degré de corrosion en fonction de la fluence

Après une exposition de 700 heures, le Z10CNT18.11 présente des piqûres et des coulures (figure 8. La qualité de tache du faisceau laser est un paramètre important pour ce type de traitement. En ef $t$, à $248 \mathrm{~nm}$, l'inhomogénéité du faisceau, notamment en bord de tache, entraîne la formation de ba. 'e de corrosion en cours des essais en brouillard salin. Avec les deux autres lasers (XeCl et LVC), il $\mathrm{r}$ a pas apparition de bande de corrosion sur les éprouvettes car les taches laser sont homogènes, ce c 'permet d'obtenir un traitement homogène sur l'ensemble de l'éprouvette.

Eche $\frac{\text { de corrosion du }}{210 \mathrm{C}}$ $\underline{\underline{Z 10 C}} \underline{\mathrm{T} 18-11}$

1 : af urition de premières piqûre:

2 : multiplication des piqûres

3 : piqûres éparses et/ou lignes de corrosion entre les bandes de balayage laser $4:$ piqûres associées à des taches étendues d'oxydes 5 : piqûres et.coulures généralisêes

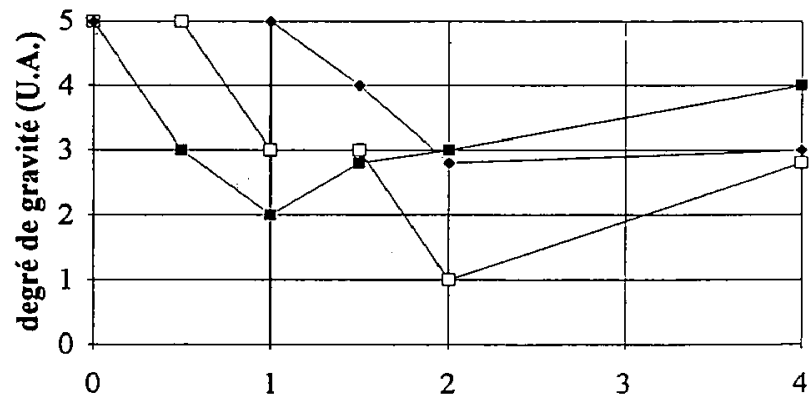

Densité d'énergie $\left(\mathrm{J} / \mathrm{cm}^{2}\right)$

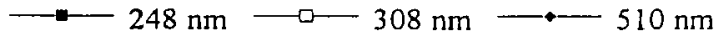

Figure 8 : Z10CNT18.11 irradié par laser, évolution de degré de corrosion en fonction de la fluence

\subsubsection{Etude tribologique : essais d'usure-frottement}

L'amélioration des propriétés tribologiques des aciers inoxydables peut se faire par de multiples traitements (dépôts, revêtements, traitements thermochimiques) adaptés à chaque configuration tribologique rencontrée.

Après traitement laser, deux tendances ont été mises en évidence pour les deux types d'inox :

- une nette diminution du coefficient de frottement pour les faibles densités d'énergie provenant de la diminution de la rugosité superficielle de l'éprouvette (figure 9),

- une augmentation du nombre de cycles pour un traitement à forte densité d'énergie $\left(4 \mathrm{~J} / \mathrm{cm}^{2}\right)$ (figure 10) provenant d'une part de l'épaisseur de la zone affectée thermiquement (environ 10 
$\mu \mathrm{m})$ et d'autre part du type d'oxyde créé à la surface de l'inox. Il existe une nette différence entre les résultats obtenus avec le laser $\mathrm{KrF}$ et les deux autres lasers ( $\mathrm{LVC}$ et $\mathrm{XeCl}$ ). En effet, le nombre de cycle est multiplié par 10 ou 20 pour le laser KrF.
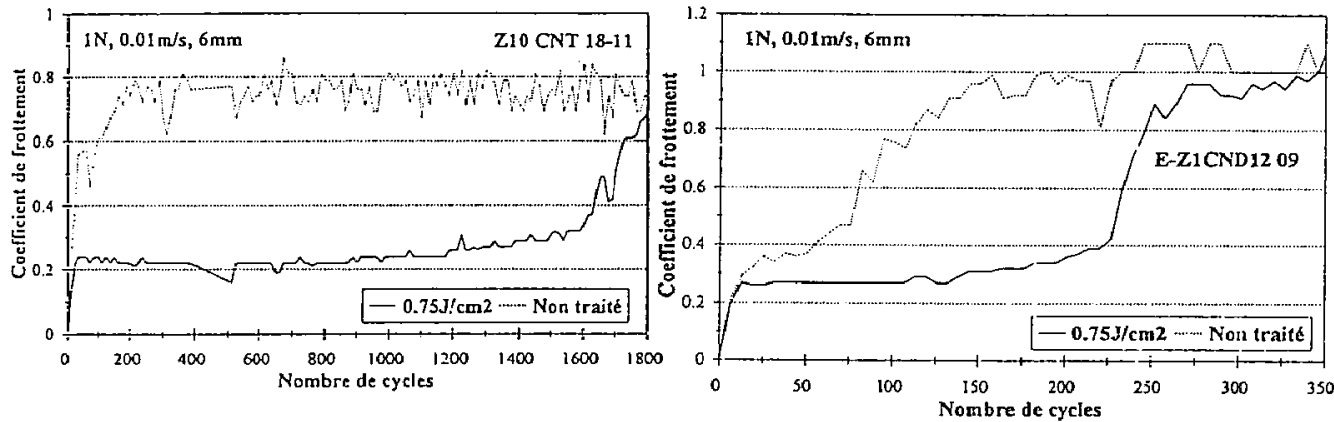

Figure 9 : évolution du coefficient de frottement pour une éprouvette traitee à $0,75 \mathrm{~J} / \mathrm{cm} 2$
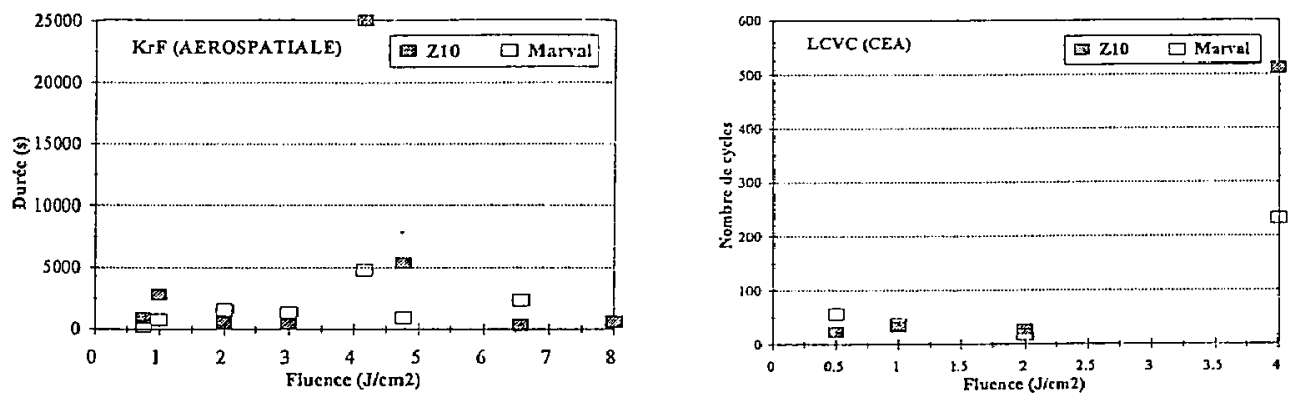

Figure 10 : évolution du nombre de cycles pour une éprouvette en inox traitée avec le laser KrF ou le LVC

\subsubsection{Analyses de surface}

Les analyses de surface par microsonde réalisées sur l'inox Z10CNT18.11, traité par laser $\mathrm{KrF}$, ont permis de mettre en évidence la présence de deux oxydes :

- un oxyde de chrome $\mathrm{Cr}_{2} \mathrm{O}_{3}$ (favorable à la résistance à la corrosion car la couche formée est dense et compacte)

- un oxyde mixte $\mathrm{NiFe}_{2} \mathrm{O}_{4}$ (défavorable à la résistance à la corrosion car la couche formée est poreuse)

Ces oxydes sont présents sur des épaisseurs d'environ 1000 à $1500 \AA$. La présence simultanée de ces deux oxydes provient de la répartition spatiale énergétique du laser qui n'est pas homogène et a recouvrement entre les taches laser. Ces deux oxydes conduisent localement à des mécanismes de résistance à l'usure ou à la corrosion différents.

\subsection{Traitement de la fonte}

Ce matériau est utilisé couramment en fonderie pour la réalisation de pièces flottantes dans les moteurs automobiles (arbre à cames, chemise de cylindres, vilebrequins, ...). De manière générale, la rectification des pièces entraîne une déformation de la matrice en fonte et un étalement en fine écaille qui peuvent être éliminer par polissage ou attaque chimique. Le traitement par laser excimère ou LVC permet une refusion de la surface qui cicatrise la surface, crée une microstructure favorable à la résistance à l'usure et découvre les nodules de graphite qui participent ainsi à l'amélioration du 
frottement. Pour des fluences supérieures à $2 \mathrm{~J} / \mathrm{cm}^{2}$, le traitement est efficace et reproductible. Le nombre de cycles est, après traitement laser, multiplié par 20 et le coefficient de frottement fortement diminué (figure 11).

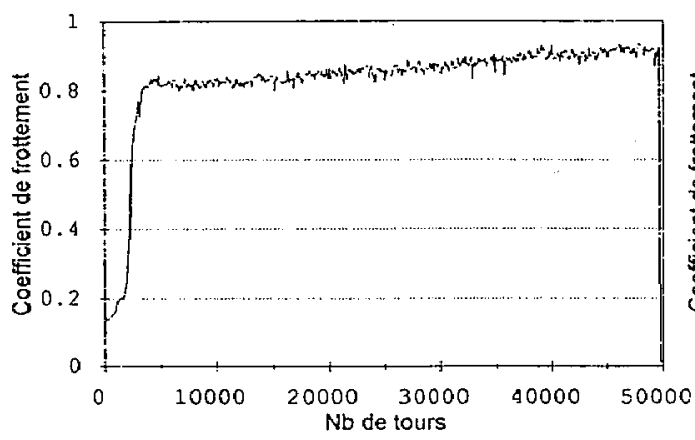

non traité

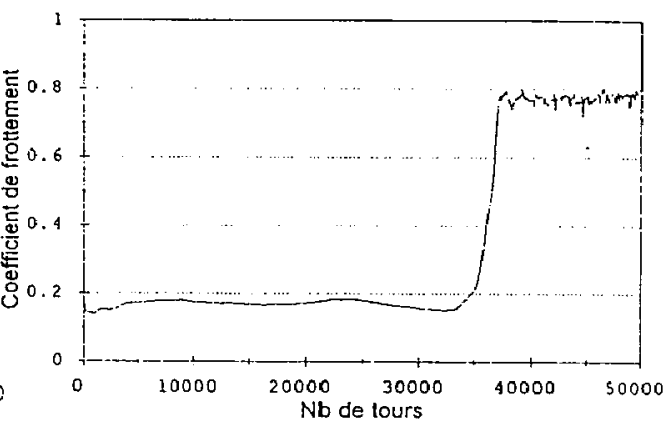

traité par laser $\left(248 \mathrm{~nm}, 3,6 \mathrm{~J} / \mathrm{cm}^{2}\right.$, recouvrement $75 \%$ )

Figure 11 : évolution du coefficient de frottement en fonction du nombre de cycle pour une éprouvette en fonte

A. Fonte a montré dans sa thèse [Refusion micrométrique superficielle par laser à exciplexes de la matrice de fonte pour l'amélioration des propriétés tribologiques, 1995], que ce type de traitement laser entraîne une fusion superficielle de la matrice et une sublimation du graphite. Il y a donc mise à nu du graphite (autolubrification) et création de bourrelet autour des éléments de graphite (protection contre l'usure). Les résultats obtenus permettent actuellement de réaliser le traitement de pièces réelles (chemise de carter) puis des tests sur banc moteur. Cette phase de l'étude est en cours.

\section{CONCLUSION}

Cette étude exploratoire a permis de démontrer les potentialités du laser excimère ou du laser à vapeur de cuivre pour la reformation de surface de matériaux. Ce type de traitement permet d'améliorer, de façon plus ou moins importante, certaines propriétés des matériaux métalliques (résistance à l'usure ou à la corrosion).

Il a été ainsi montré que par rapport aux matériaux non traités :

- pour l'alliage d'aluminium Al2024, une amélioration de la tenue à la corrosion pour des densités d'énergie supérieures à $1,5 \mathrm{~J} / \mathrm{cm}^{2}$,

- pour le Marval X12, une nette amélioration de la résistance au frottement notamment pour les longueurs d'onde du laser excimère mais une forte dégradation de la résistance à la corrosion

- pour le Z10CNT18.11, une amélioration de la résistance au frottement et à la corrosion,

- pour l'AS7, une légère amélioration de la résistance au frottement.

Toutefois les premiers résultats obtenus restent très en deçà des traitements de référence utilisés à ce jour et ne peuvent être concurrentiels au niveau industriel.

Pour la fonte, il a été observé une très nette amélioration des propriétés tribologiques après traitement laser. La validation de ce traitement sur pièce réel est en cours.

\section{Remerciements}

Ces travaux ont été réalisés en collaboration avec PSA (direction des Méthodes et moyens industriels, $M$. Frainais), le CEA (direction du cycle du combustible, J.M. Weulerse et J. Maury), l'IRPHE (laboratoire LP3, M.Sentis) et l'ECole des Mines de Paris (Centre des matériaux P.M. Fourt, $M$. Jeandin). Le contrat est géré par SYLAREC (J.P. Gex). Ces études ont été soutenues par le MRES, le MIPTCE (EU205), PSA et AEROSPATIALE. 